\title{
Singularity Problem with the One-sheet Huygens Subgridding Method
}

DOI:

10.1109/TEMC.2016.2624511

\section{Document Version}

Accepted author manuscript

Link to publication record in Manchester Research Explorer

\section{Citation for published version (APA):}

Al-Tameemi, H., Bérenger, J. P., \& Costen, F. (2017). Singularity Problem with the One-sheet Huygens Subgridding Method. IEEE Transactions on Electromagnetic Compatibility, 5(3), 992-995.

https://doi.org/10.1109/TEMC.2016.2624511

\section{Published in:}

IEEE Transactions on Electromagnetic Compatibility

\section{Citing this paper}

Please note that where the full-text provided on Manchester Research Explorer is the Author Accepted Manuscript or Proof version this may differ from the final Published version. If citing, it is advised that you check and use the publisher's definitive version.

\section{General rights}

Copyright and moral rights for the publications made accessible in the Research Explorer are retained by the authors and/or other copyright owners and it is a condition of accessing publications that users recognise and abide by the legal requirements associated with these rights.

\section{Takedown policy}

If you believe that this document breaches copyright please refer to the University of Manchester's Takedown Procedures [http://man.ac.uk/04Y6Bo] or contact uml.scholarlycommunications@manchester.ac.uk providing relevant details, so we can investigate your claim.

\section{OPEN ACCESS}




\title{
Singularity Problem with the One-sheet Huygens Subgridding Method
}

\author{
Hayder AL-Tameemi, Student Member, IEEE, Jean-Pierre Bérenger, Fellow IEEE, Fumie Costen, Senior \\ Member, IEEE,
}

\begin{abstract}
The Huygens subgridding (HSG) relies on the connection of two FDTD grids by means of Huygens surfaces. An earlier work has presented that the Huygens surfaces can be reduced to one sheet of current in place of the usual two sheets. This letter shows that the resulting simplified HSG may be at the origin of inaccuracy in the computed results because of the singularity of the field at edges of perfect electric or magnetic conductors. The difficulty can be easily overcome in two dimensions but not easily in three dimensions where it is preferable to use the one sheet implementation only for the outer Huygens surface.
\end{abstract}

Index Terms-Maxwell equations, FDTD, subgridding, Huygens surface.

\section{INTRODUCTION}

$\mathrm{T}^{\mathrm{H}}$ HE Huygens Subgridding (HSG) technique [1] $-[3]$ relies on the connection of two Finite-Difference Time-Domain (FDTD) grids by means of Huygens surfaces [4]. The Inner Surface (IS) radiates the field from the main grid into the subgrid, and the Outer Surface (OS) radiates the field from the subgrid into the main grid. The set composed of IS, OS and the two grids is equivalent to the physical problem to be solved [1], [3]. The method has some advantages, such as the possibility of using large ratios of FDTD steps. It may suffer from late time instability in some cases. This drawback can be removed by using an unconditionally stable method in the subgrid, for instance the ADI-FDTD [5].

The implementation of the HSG in a computer code is complex, which is a feature it shares with most subgridding techniques. It is thus highly desirable to simplify it as much as possible. Such a simplification has been proposed in [3]. While usually a Huygens surface [4] requires impressing both electric and magnetic current sheets, in the case of the HSG one sheet of IS or OS can be removed. This is possible because the principle of the HSG involves two non-physical regions refereed to as the non-working regions [1], [3]. They can be replaced with either a Perfect Electric Conductor (PEC) or a Perfect Magnetic Conductor (PMC). This forces one of the two sheets of IS and OS to vanish, which in turn results in one-sheet IS and OS. The encoding of the HSG is thus halved whilst the equivalence of the numerical problem to the initial physical problem is preserved. We denote the simplified HSG [3] as the one-sheet HSG.

Hayder AL-Tameemi, Jean-Pierre Bérenger, Fumie Costen are with the School of Electrical and Electronic Engineering, The University of Manchester, U.K. (email: fumie.costen@manchester.ac.uk)

Color versions of the figures in this paper are available online at http://ieeexplore.iee.org.
Although the one-sheet HSG and the two-sheet HSG are in theory equivalent, we show in this letter that in practice the accuracy of the one-sheet HSG may be a little lower. This is due to the singularity of the $\boldsymbol{E}$ or $\boldsymbol{H}$ fields at the edges of the PEC or PMC which replace the non-working region of the main grid. This renders the Inner Surface (IS) inaccurate. The problem is demonstrated by a significantly wider range of investigations than in previous studies [3]. We show numerical simulations which demonstrate the order of magnitude of the errors which may result from the singularity problem. In two dimensional (2D) computational domains, the singularity problem can be removed by an adequate choice of the location of IS, rendering the one-sheet HSG as accurate as the two-sheet HSG. However, in 3D, there is no evident and simple solution to this question.

\section{The Singularity PROBLEM WITH THE ONE-SHEET HSG}

We demonstrate the problem which results from the singularity of the field near a PEC or PMC edges in the 2D $\mathrm{TM}_{z}$ case which has $\boldsymbol{H}_{\boldsymbol{x}}, \boldsymbol{H}_{\boldsymbol{y}}, \boldsymbol{E}_{\boldsymbol{z}}$. Parts of the main grid and subgrid are depicted in Fig. 11 where IS radiates the field from the main grid to the subgrid. In Fig. 1(a), IS is placed aside a PEC whilst in Fig. 1(b) it is aside a PMC. In both cases, either the PEC or the PMC replaces the non-working region of the main grid [1] $-[3]$. We use the same notations and variables as the ones used in [1]-[3].

With the PEC in Fig. 1 (a), the implementation of the $y$ directed part of IS consists of correcting the FDTD advance of $E_{b z}$ at nodes $i b$ of the subgrid using $H_{a y}^{*}$ on fictitious $i a^{*}$ of the main grid [2], [4]. This can be written, omitting index the in $y$-direction as

$$
\begin{aligned}
& E_{b z}^{n+1}(i b)=E_{b z}^{n}(i b) \\
& -\frac{\Delta t_{b}}{\epsilon_{0} \Delta x_{b}}\left(H_{b y}^{n+\frac{1}{2}}(i b) \quad-H_{b y}^{n+\frac{1}{2}}(i b-1)+H_{a y}^{*}\left(i a^{*}\right)\right),
\end{aligned}
$$

where $\Delta t_{b}$ and $\Delta x_{b}$ are the steps in the subgrid, $i a$ and $i b$ are the IS location in the main and subgrid respectively and $H_{a y}^{*}\left(i a^{*}\right)$ is the fictitious field set equal to the field $H_{a y}$ at node $i a-\frac{1}{2}$ (with interpolation in $y$-direction for the fictitious nodes absent at $i a-\frac{1}{2}$ ). No correction is applied to the advance of $H_{b y}$ at $i b-\frac{1}{2}$ because $E_{a z}$ at $i a$ is zero (zero current sheet). What should be noticed is that the component impressed as a current sheet in the subgrid, i.e. $H_{a y}$, is perpendicular to the $z$-directed edge of PEC which is reduced to a corner in the $2 \mathrm{D}$ grid. This $H_{a y}$ is proportional to the density of the 
surface current flowing in direction parallel to the edge and the density tends to infinity when the distance from the edge vanishes. More precisely, from [6], [7], $H_{a y}$ can be written as

$$
H_{a y}(r, \theta)=\frac{A(\theta)}{r^{\frac{1}{3}}},
$$

where $r$ and $\theta$ are polar coordinates centered at the edge which is $E_{a z}$ node at the corner of the PEC in Fig. 11 a). $A(\theta)$ is a coefficient from [6], [7]. In the case of the implementation of IS in Fig. 1 b), $H_{b y}(i b)$ must be corrected with a fictitious $E_{a z}^{*}\left(i a^{*}\right)$, i.e. advanced with an equation in the form of

$$
\begin{aligned}
& H_{b y}^{n+\frac{1}{2}}(i b)=H_{b y}^{n-\frac{1}{2}}(i b) \\
& -\frac{\Delta t_{b}}{\mu_{0} \Delta x_{b}}\left(E_{b z}^{n+1}(i b+1)-E_{b z}^{n+1}(i b)+E_{a z}^{*}\left(i a^{*}\right)\right) .
\end{aligned}
$$

Here, the component impressed as a current sheet $E_{a z}$ is parallel to the edge of PMC, which means that it is not singular. This is an essential difference from the implementation in Fig. 11(a), as demonstrated in the following.

Concerning the Outer Surface (OS) which radiates the field from the subgrid to the main grid, there is no singularity near the edges of the PEC or PMC which replace the non-working region of the subgrid. This is because the wedges formed by the two PEC or PMC walls are re-entrant. In this case, it is known that both $\mathrm{E}$ and $\mathrm{H}$ fields are not singular [6], [7]. Therefore it is expected that the one-sheet OS remains as accurate as the two-sheet one. This was confirmed by numerical simulations as the one reported in Section III.

Fig. 2 depicts a 2D $\mathrm{TM}_{z}$ numerical simulation scenario where the surface IS is $4 \times 50$ cells in size in the main grid. The ratio of steps of the two grids $n s$ is 5 . An incident wave propagating in $x$ direction is generated in the main grid and its waveform is the Gaussian pulse $H_{y}(t)=100 e^{-\frac{t-3 \theta}{\theta}}$ $\mathrm{A} / \mathrm{m}$, where $\theta=20 \Delta t_{a}$. Fig. 3 shows $H_{y}$ at four observation nodes in the subgrid, computed with implementations of IS in Fig. 11a) and Fig. 11b). No object is present in both grids which means that the field should be a copy of the incident field. The $\boldsymbol{H}_{\boldsymbol{y}}$ field with a PEC non-working region (Fig. 3 (a)) agrees with the incident field (magnitude $100 \mathrm{~A} / \mathrm{m}$ ) at node 4 in the middle of the IS region, but as the observation approaches the upper corners of IS the magnitude of the pulse decreases. At observation node 1, the error is about $30 \%$. On the other hand, with the PMC non-working region (Fig. 3 b)), the field matches the incident field regardless of the observation location in the subgrid. The results in Fig. 3 are in close agreement with what was expected. With the PEC implementation in Fig. 11 a) the $H$ component involved in the sheet of IS is singular at the corner, resulting in a significant error in the estimates used to compute $H_{a x}^{*}$ and $H_{a y}^{*}$ radiated into the subgrid. In the case of the PMC implementation in Fig. 11.b) the $E_{a z}$ component used in the sheet of IS is parallel to the edge of PMC and thus no significant error is produced.

Fig. 4 shows results of a numerical simulation with the same simulation settings as in Fig. 2, but with a rectangular PEC object of size $10 \times 230$ cells in the subgrid. Component $H_{y}$ at node 3 in Fig. 2, which is at the top of the PEC object, is compared with a reference solution computed within a single FDTD domain with the same mesh as that of the subgrid.

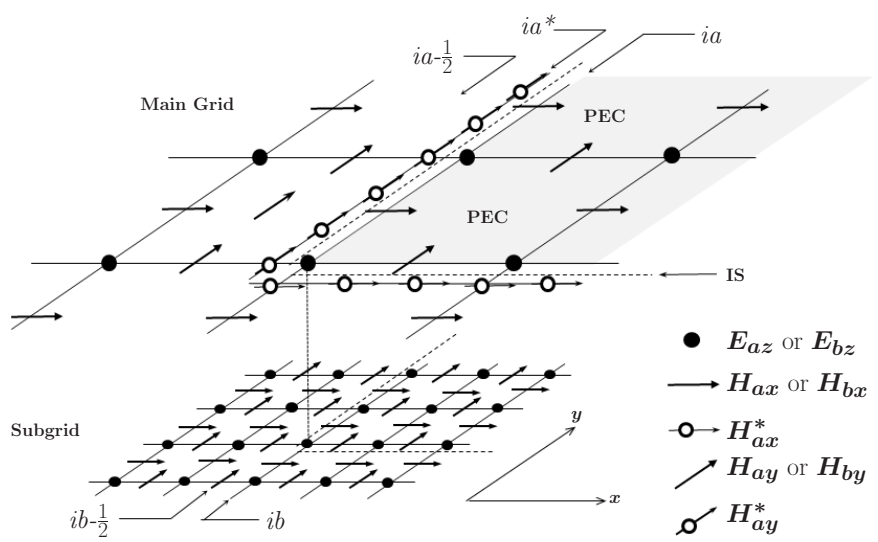

(a)

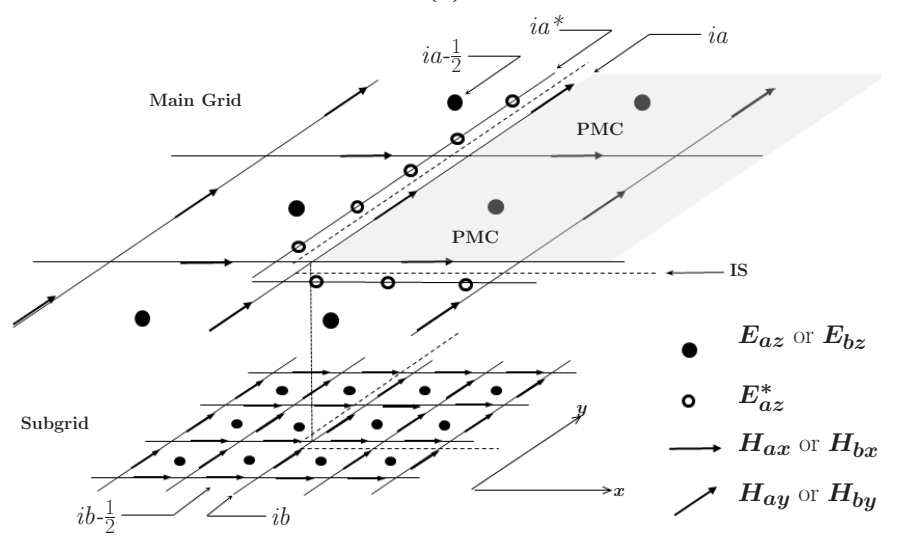

(b)

Fig. 1. The two implementations of the one-sheet IS in the $2 \mathrm{D} \mathrm{TM} z$ case. (a) with the non-working region replaced with a PEC, (b) with the non-working region replaced with a PMC. Nodes $\boldsymbol{E}_{\boldsymbol{a} z}^{*}, \boldsymbol{H}_{\boldsymbol{a x}}^{*}, \boldsymbol{H}_{\boldsymbol{a} \boldsymbol{y}}^{*}$ are fictitious nodes where estimates of $\boldsymbol{E}_{\boldsymbol{z}}, \boldsymbol{H}_{\boldsymbol{x}}, \boldsymbol{H}_{\boldsymbol{y}}$ are needed to impress the current sheet of IS in the subgrid.

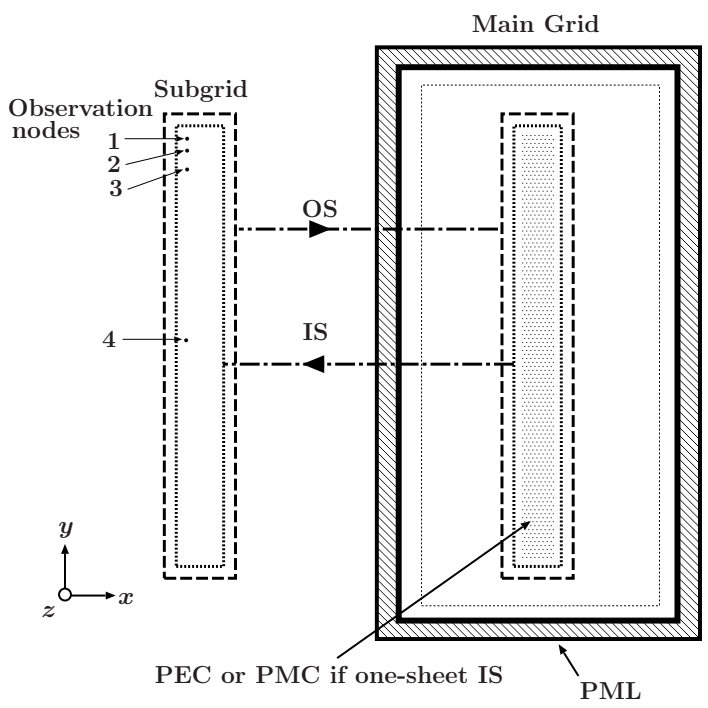

Fig. 2. A $2 \mathrm{D} \mathrm{TM} z$ numerical simulation scenario with main grid steps $1 \mathrm{~mm}$ and $1.9 \mathrm{ps}$. The gap between OS and IS is 3 main grid steps. The size of IS is $4 \times 50$ main cells and $20 \times 250$ sub cells. Nodes $1,2,3,4$ are $2,5,10,125$ subcells from the top of IS, respectively, and 4 subcells from the left-hand side of IS. In the numerical simulations reported in Fig. 4 a $10 \times 230$-cell object is placed within IS in the subgrid. 


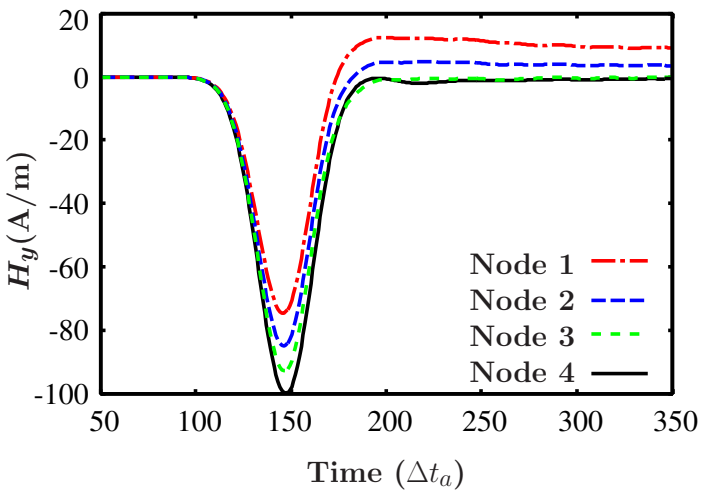

(a)

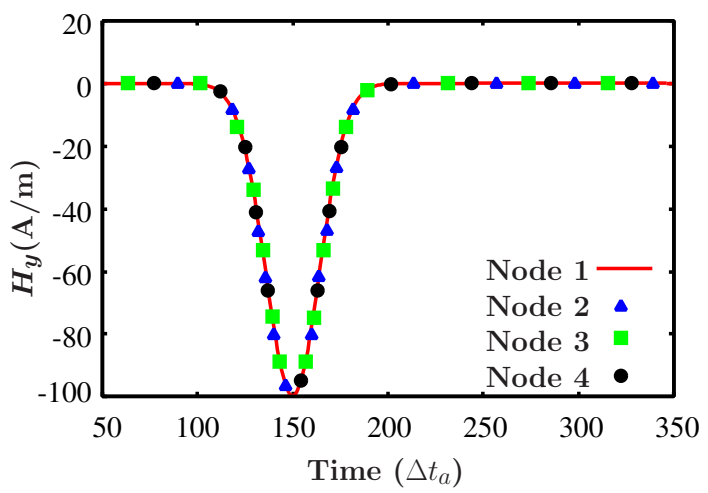

(b)

Fig. 3. $\boldsymbol{H}_{\boldsymbol{y}}$ field at the nodes $1,2,3$ and 4 of the subgrid shown in Fig. 2 Results were computed with the non-working region of the main grid replaced with either (a) a PEC (Fig. 1 a)) or (b) a PMC (Fig. 1 b b)). The one-sheet OS was used in both numerical simulations.

Fig. 4 shows that the HSG result computed with a PMC non-working region in the main grid agrees very well with the reference solution, while the result computed with a PEC non-working region is significantly erroneous because of the singularity of the field used in the Huygens sheet.

The $2 \mathrm{D} \mathrm{TE}_{z}$ case, with components $E_{x}, E_{y}$ and $H_{z}$, is the dual of the $\mathrm{TM}_{z}$ case. As is the $2 \mathrm{D} \mathrm{TM}_{z}$ case we have both PMC and PEC implementations. Numerical simulations yield similar results as in the $\mathrm{TM}_{z}$ case. The situation is reversed, i.e. in the $\mathrm{TE}_{z}$ case the singularity is present when using a PMC non-working region. It can be removed by using a PEC non-working region.

\section{3D NUMERICAL SIMULATIONS}

Fig. 5 reports the results of the numerical simulation for a problem similar to the one in Fig. 2, with a PEC object inside the subgrid. The simulation settings are identical to those in [Fig. 9, 3], with a $1 \times 1 \times 150$-cell object in the subgrid. With the one-sheet IS and OS, the non-working regions are filled with a PEC in the main grid and a PMC in the subgrid, as in [3]. The $E_{x}$ and $E_{z}$ components normal to the physical interface between the air and the PEC object (same observation location as in [3]) are plotted in Fig. 5 In addition to the reference solution in [3], three results are reported in the figure. The first one is computed with the one-sheet IS and the two-

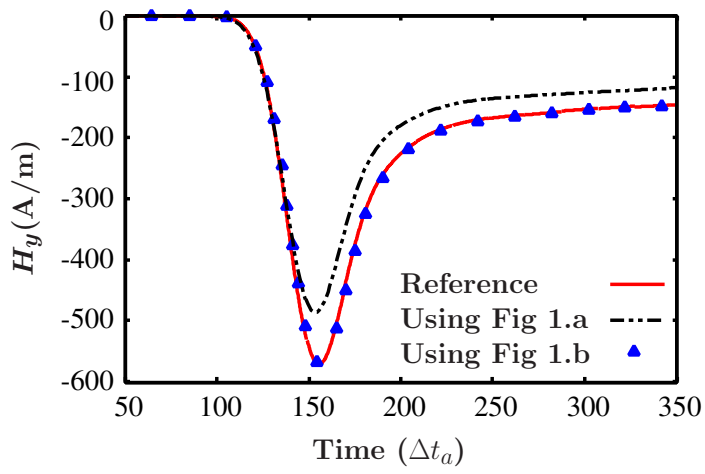

Fig. 4. $\boldsymbol{H}_{\boldsymbol{y}}$ field at the top of a $10 \times 230$-cell PEC object in the subgrid in Fig. 2 computed with either a PMC or a PEC in the non-working region of the main grid. The one-sheet OS was used in both numerical simulations.

TABLE I

ERROR PERCENTAGE CALCULATED FROM FIG.5

\begin{tabular}{cc}
\hline HSG & Error \\
\hline One-sheet IS and Two-sheet OS & $13.1 \%$ \\
Two-sheet IS and One-sheet OS & $0.32 \%$ \\
One-sheet IS and One-sheet OS & $11.5 \%$ \\
\hline
\end{tabular}

sheet OS, the second one with the two-sheet IS and the onesheet OS, and the third one with both the one-sheet IS and the one-sheet OS.

As is observed, the calculation with the one-sheet IS significantly disagrees with the reference solution. On the other hand, the one-sheet OS with two-sheet IS does not degrade the accuracy of the results. With the one-sheet IS and the one-sheet OS the results are intermediate. The presence of the PMC of the one-sheet OS reduces the spurious effect of the one-sheet IS, but the results remain unacceptable.

In $3 \mathrm{D}$ as well as in $2 \mathrm{D}$, it is possible to fill the nonworking region of the main grid with either a PEC, as in the simulation in Fig. 5, or with a PMC. However, the components used to produce the one-sheet IS are always perpendicular to the edges. These are $\boldsymbol{H}$ components with a PEC region or $\boldsymbol{E}$ components with a PMC region. In both cases the components used in IS are then singular, which means that the field radiated into the subgrid suffers from inaccuracy. Contrary to the $2 \mathrm{D}$ case where the problem can be removed by using the adequate location of IS, there is no trivial solution to solve the singularity problem in 3D. There is thus a significant irreducible error when using both the one-sheet IS and the onesheet OS. However, a combination of the two-sheet IS with the one-sheet OS preserves accuracy, as demonstrated in Fig. 5 Table $\mathrm{I}$ shows the error of each type of the HSG in Fig.5. The error is quantified by calculating the difference between each HSG method and the reference solution using [(10), [8]].

The difference of the computational time of the four calculations in Fig. 5 is less than 1\%. As stated in the introduction the objective of the one-sheet HSG is the reduction of the complexity of encoding, not the reduction of computational time which may be a few percent in the best case. 


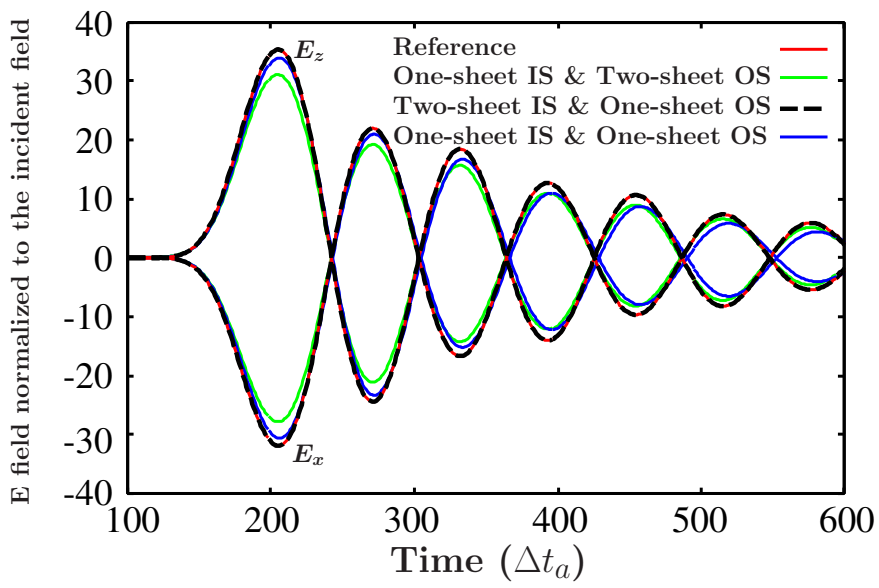

Fig. 5. Comparison of the one-sheet HSG with the conventional two-sheet HSG for the 3D numerical simulation defined in [Fig. 9, 3]. The plotted $E_{x}$ and $E_{z}$ components are perpendicular to the surface of the PEC wire at its end.

\section{CONCLUSION}

The one-sheet HSG proposed in [3] to reduce the encoding effort of the HSG method may suffer from inaccuracy of the field radiated by the Inner Huygens Surface into the subgrid. The inaccuracy is due to the singularity of the field at the edges of the PEC or PMC with which the non-working region of the main grid is replaced. In 2D the singularity can be removed by using a PEC non-working region in the $\mathrm{TE}_{z}$ case and a PMC non-working region in the $\mathrm{TMz}$ case, rendering the one-sheet HSG effective. In 3D the singularity is present with both PEC and PMC non-working regions in the main grid. Reduction of the effect of the singularity may be possible [9], but this would reduce the interest of the one-sheet HSG, whose main purpose is the reduction of the encoding effort in comparison with the normal two-sheet HSG. However, a combination of a one-sheet Outer Surface (OS) with a two-sheet Inner Surface (IS) is possible in $3 \mathrm{D}$.

\section{REFERENCES}

[1] J.-P. Bérenger, "A Huygens subgridding for the FDTD method," IEEE Trans. Antennas Propag., vol. 54, no. 12, pp. 3797-3804, Dec. 2006.

[2] — , "Extension of the FDTD Huygens subgridding algorithm to two dimensions," IEEE Trans. Antennas Propag., vol. 57, no. 12, pp. 38603867, Dec. 2009.

[3] — "The Huygens subgridding for the numerical solution of the Maxwell equations," J. Comput. Phys., vol. 230, pp. 5635-5659, 2011.

[4] A. Taflove and S. Hagness, Computational Electromagnetics. The FiniteDifference Time-Domain Method. Boston, MA: Artech House, 2005.

[5] Z. Huang, G. G. Pan, and K. S. Chen, "A synchronized multigrid time domain method via huygens subgridding and implicit algorithms," IEEE Trans. Antennas Propag., vol. 61, no. 5, pp. 2605-2614, May 2013.

[6] J. Van Bladel, Electromagnetic Fields, ser. Second Edition. Wiley, 2007, ch. 5, pp. 171-174.

[7] _ Singular Electromagnetic Fields and Sources. Oxford Science Publications, 1991, ch. 4, pp. 118-124.

[8] B. Abdulkareem, J. P. Bérenger, F. Costen, R. Himeno, and H. Yokota, "Huygens Excitation in Debye Media in the FDTD Method," IEEE Trans. Antennas Propag., vol. 64, no. 8, pp. 3632-3635, Aug 2016.

[9] S. M. Foroughipour and K. P. Esselle, "The theory of a singularityenhanced FDTD method for diagonal metal edges," IEEE Trans. Antennas Propag., vol. 51, no. 2, pp. 312-321, Feb 2003.

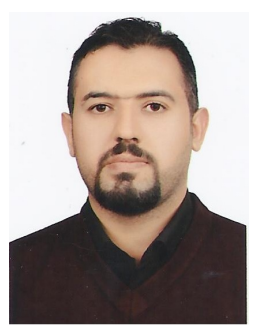

Hayder AL-Tameemi received the B.Sc. degree in information technology engineering from the University of Technology, Baghdad, Iraq in 2005 and the M.Sc. in Data communication systems from Brunel University, London, UK in 2008. He is currently working toward the Ph.D. degree in electrical and electronic engineering in the Univeristy of Manchester, Manchester, UK.

His research interests include the finite-difference time-domain methods, subgridding, dispersive materials modeling, computational electromagnetics, bioelectromagnetics, high performance computing and parallel programming languages.

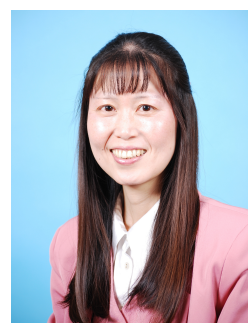

Fumie Costen (SM'12) received the B.Sc. degree, the M.Sc. degree in electrical engineering and the $\mathrm{Ph} . \mathrm{D}$. degree in Informatics, all from Kyoto University, Japan.

From 1993 to 1997 she was with Advanced Telecommunication Research International, Kyoto, where she was engaged in research on direction-ofarrival estimation based on Multiple SIgnal Classification algorithm for 3-D laser microvision. She filed three patents from the research in 1999 in Japan. She was invited to give 5 talks in Sweden and Japan during 1996-2014. From 1998 to 2000, she was with Manchester Computing in the University of Manchester, U.K., where she was engaged in research on metacomputing and has been a Lecturer since 2000. Her research interests include computational electromagnetics in such topics as a variety of the finite difference time domain methods for microwave frequency range and high spatial resolution and FDTD subgridding and boundary conditions. She filed a patent from the research on the boundary conditions in 2012 in the U.S.A. Her work extends to the hardware acceleration of the computation using general-purpose computating on graphics processing units, Streaming Single Instruction Multiple Data Extension and Advanced Vector eXtentions instructions. Dr. Costen received an ATR Excellence in Research Award in 1996 and a best paper award from 8th International Conference on High Performance Computing and Networking Europe in 2000.

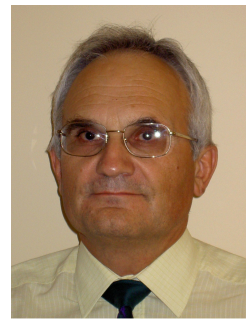

Jean-Pierre Brenger (F09) received a Master in Physics from the University Joseph Fourier, Grenoble, France, in 1973, and a Master in Optical Engineering from the Institut dOptique Graduate School, Paris, France, in 1975

From 1975 to 2013 he was with the Direction Gnrale de 1Armement of France, where he was a research engineer on the electromagnetic effects of nuclear rays (1975-1989), he held a position as expert on the electromagnetic effects of nuclear events (1989-1998), and he was a contract manager while staying active in the field of numerical electromagnetic (1998-2013). He is currently a consultant on electromagnetic effects of nuclear rays and on numerical electromagnetics, and a visiting professor at the School of Electrical and Electronic Engineering, the University of Manchester, UK. His researches include low frequency propagation, absorbing boundary conditions, and FDTD method. He is a member of the Electromagnetics Academy and has been an Associate Editor of the IEEE Transactions on Antennas and Propagation from 2006 to 2010. He received the 2013 Medal of URSI-France and the 2014 John Howard Dillinger Gold Medal from URSI. 